\title{
Prognostic Biomarkers at Discontinuation of Renal Replacement Therapy in Acute Kidney Injury Patients in the Intensive Care Unit
}

\author{
Tingting Yang Yuliang Zhao Qiang Liu Mei Han Si Sun Liping Lin Baihai Su \\ Songmin Huang
}

Department of Nephrology, West China Hospital, Sichuan University, Chengdu, Sichuan, PR China

Dear Editor,

There remains uncertainty about the optimal timing of discontinuing renal replacement therapy (RRT) in acute kidney injury (AKI) patients in the intensive care unit (ICU) [1]. Conventional indicators such as serum creatinine (sCr) and urine output (UO) may be susceptible and not accurate enough to reflect the progress or repair. Biomarkers are involved in the pathogenesis of AKI [2]. Sensitive and specific novel biomarkers at an early stage of AKI have been reported to be early predictors of the need for RRT [3]. Nevertheless, the predictive values of biomarkers at the time of discontinuing RRT for mid-term survival-related successful weaning of RRT are unknown.

To seek biomarker reference indicators for the discontinuation of RRT, we performed a preliminary prospective observational study in the ICUs of West China Hospital of Sichuan University, China. The study was approved by the local ethics Committee and written informed consent was obtained from participants. The study enrolled 42 RRT-requiring AKI patients who weaned and remained independent from RRT or died without requirement of acute re-initiation. RRT was stopped when azotemia, oliguria, acidosis and hyperkalemia were significantly improved. In order to reduce the possible influence of clearance effect of RRT, blood samples were

Table 1. AUC for biomarkers on discontinuation of RRT for predicting survival

\begin{tabular}{lll}
\hline Biomarkers & AUC & $95 \%$ CI \\
\hline sOPN & 0.809 & $0.630-0.988$ \\
sIL-6 & 0.724 & $0.490-0.959$ \\
sIL-18 & 0.616 & $0.430-0.802$ \\
sNGAL & 0.566 & $0.332-0.800$ \\
sCysC & 0.537 & $0.331-0.742$ \\
UO & 0.601 & $0.415-0.787$ \\
sCr & 0.484 & $0.276-0.692$
\end{tabular}

All variables were inversely predictive for survival except UO.

$\mathrm{s}=$ Serum; NGAL = neutrophil gelatinase-associated lipocalin; $\mathrm{CysC}=$ cystatin $\mathrm{C}$.

obtained $16-48 \mathrm{~h}$ after the discontinuation of RRT. The levels of serum osteopontin (sOPN), interleukin-6 (IL-6), IL-18, neutrophil gelatinase-associated lipocalin and cystatin $\mathrm{C}$ were measured by sandwich enzyme-linked immunosorbent assay or immunoturbidimetric assay. Patients were followed up for 90-day survival.

Eleven (26.2\%) patients died during follow-up. The mean levels of all biomarkers were higher in death group. Diabetes, Charlson comorbidity index, sepsis, APACHE II score, all biomarkers, sCr and UO were included in the primary logistic regression analysis. By the enter method, the final model identified no sepsis (OR
0.005; 95\% CI $0.001-0.637 ; \mathrm{p}=0.032)$, no diabetes (OR 0.011; 95\% CI 0.001-0.593; $\mathrm{p}=0.027)$, lower APACHE II score (OR $0.641 ; 95 \%$ CI $0.416-0.985 ; \mathrm{p}=0.043)$ and lower sOPN on discontinuation of RRT (OR 0.962; 95\% CI 0.926-1.000; $\mathrm{p}=0.048$ ) as predictors for 90 -day survival. The area under the receiver operating characteristic curve (AUC) of all biomarkers, $\mathrm{sCr}$ and $\mathrm{UO}$ for survival is shown in table 1 . Lower sOPN reached the highest AUC of 0.809 $(\mathrm{p}=0.003)$ and lower serum IL-6 (sIL-6) also performed better than others (AUC $0.724 ; \mathrm{p}=0.029)$. Generally, biomarkers showed better ability for predicting survival.

\section{KARGER}

E-Mail karger@karger.com

www.karger.com/bpu (c) 2016 S. Karger AG, Base

0253-5068/16/0424-0347\$39.50/0
Baihai Su

Department of Nephrology, West China Hospital

Sichuan University, No. 37 Guo Xue Xiang

Chengdu, Sichuan 610041 (PR China)

E-Mailimsbh@163.com 
Our preliminary study found that lower sOPN showed best predictive ability for 90-day survival and lower sIL-6 also showed moderately predictive ability, which performed better than $\mathrm{sCr}$ and UO. Other than short-term re-initiation of RRT and mortality, mid-to-long-term mortality should also be considered when making decisions of withdrawing RRT. The expression of OPN in tubule segments and glomeruli is significantly increased when stimulated by damage. OPN acts as a protective factor that increases cell tolerance to ischemia and hypoxia, suppressing cell apoptosis and has a role in the process of tubular epithelial cells regeneration after acute injury [4]. OPN also plays a pro-inflammatory role in sepsis and predicts mortality [5]. Lorenzen et al. [6] found that patients who had been successfully weaned from RRT showed significantly decreased OPN level
( $\mathrm{p}=0.0005)$ during RRT and OPN was not cleared by dialysis. All the findings indicate that lower OPN was a stable and good predictor for survival. IL-6 can promote inflammatory reaction and reduce oxidation at the same time in renal tissue [7]. The finding that sOPN and sIL- 6 showed better predictive abilities might be attributed to the fact that sOPN and sIL6 levels were more associated with systemic inflammatory response, which correlated with death. In addition, other biomarkers may have higher clearance coefficients during RRT making them less predictive for survival. Uchino et al. [8] found that UO predicted best for re-RRT requirement within 7 days, while we find UO predicted poorly for 90day survival. What they focused on was short-term improvement of renal function. UO may have better predictive ability for renal outcome rather than survival.
In conclusion, we found that lower sOPN at the time of discontinuation of RRT independently predicted 90-day survival and lower sIL-6 also performed well. Lower sOPN and sIL-6 may be reference indicators of withdrawing RRT based on conventional indicators in critically ill patients. Larger and more rigorous study is needed to further explore this issue.

\section{Acknowledgement}

The study was funded by Key Program of National Nature Science Foundation of China (51433007-1), Plan Projects of Science and Technology of Sichuan Province (2014SZ0029) and Chengdu Peoplebenefit Technology Program (2014-HM0100323-SF).

\section{References}

1 Gibney RT, Bagshaw SM, Kutsogiannis DJ, Johnston C: When should renal replacement therapy for acute kidney injury be initiated and discontinued. Blood Purif 2008;26:473484.

2 Lisowska-Myjak B: Serum and urinary biomarkers of acute kidney injury. Blood Purif 2010;29:357-365.

3 Gonzalez F, Vincent F: Biomarkers for acute kidney injury in critically ill patients. Minerva Anestesiol 2012;78:1394-1403.
4 Xie Y, Sakatsume M, Nishi S, Narita I, Arakawa M, Gejyo F: Expression, roles, receptors, and regulation of osteopontin in the kidney. Kidney Int 2001;60:1645-1657.

5 Fortis S, Khadaroo RG, Haitsma JJ, Zhang H: Osteopontin is associated with inflammation and mortality in a mouse model of polymicrobial sepsis. Acta Anaesthesiol Scand 2015;59: 170-175.

6 Lorenzen JM, Hafer C, Faulhaber-Walter R, Kümpers P, Kielstein JT, Haller H, Fliser D: Osteopontin predicts survival in critically ill patients with acute kidney injury. Nephrol Dial Transplant 2011;26:531-537.
7 Nechemia-Arbely Y, Barkan D, Pizov G, Shriki A, Rose-John S, Galun E, Axelrod JH: IL-6/ IL-6R axis plays a critical role in acute kidney injury. J Am Soc Nephrol 2008;19:1106-1115.

8 Uchino S, Bellomo R, Morimatsu H, Morgera S, Schetz M, Tan I, Bouman C, Macedo E, Gibney N, Tolwani A, Straaten HO, Ronco C, Kellum JA: Discontinuation of continuous renal replacement therapy: a post hoc analysis of a prospective multicenter observational study. Crit Care Med 2009;37:2576-2582. 